\title{
Evaluationsstudie für den Zusammenhang der Textauswahl und der Textkompetenzen im Lichte der Selbsteinschätung der DaF-Studierenden an der Pädagogischen Fakultät der Helwan Vniversität
}

\author{
Ass.Prof. Duaa Abd El Karim
}

Pädagogischen Fakultät der Helwan Universität

\section{Abstract}

$D$

ie Texte sind in der DaF-Lehrerausbildung relevant und haben eine wirksame Bedeutung. Das vierjährige Studium enthält unterschiedliche germanistische und pädagogische Fächer, in denen die DaFStudierenden mit Texten arbeiten können. Die vorliegende Arbeit führt den Umgang mit Texten, der Textauswahl und der Textkompetenz in der universitären DaF-Lehrerausbildung vor. Welche Rolle können die Texte bei der Lehrerausbildung spielen? Und wie wirkt sich die Textauswahl auf die Textkompetenzen der Studierenden aus?.

Dieser Beitrag befasst sich mit den angebotenen Texten in der universitären DaF-Lehrerausbildung an der pädagogischen Fakultät der Helwan Universität in Ägypten. Die Arbeit stützt sich zuerst auf die Einstellungen und Meinungen der DaF-Studierenden über Textauswahl und danach auf die Selbsteinschätzung ihrer Textkompetenzen. Deshalb habe ich eine Befragung entwickelt, damit die Studierenden ihre Meinungen über Texte und die Textauswahl während des Studiums ausdrücken können. Die Auswertungen und Einstellungen der Studierenden werden notiert und analysiert. Dann habe ich auch eine Befragung der Selbsteinschätzung entwickelt, damit sie ihre Textkompetenzen bewerten können. Die Ergebnisse helfen dabei, ein Profil für die Textkompetenzen bei den DaF-Studierenden zu erstellen.

Am Ende werden an Hand der Ergebnisse der Befragungen und an Hand der Komponenten der universitären DaFLehrerausbildung Textauswahlkriterien vorgeschlagen und konsequent eingesetzt.

Schlagwörter: Evaluationsstudie - Text - Textkompetenz Textauswahlkriterien - DaF-Lehrerausbildung.

\section{1-Einleitung}

Das Sprachenlernen wird auf Texte aufgebaut. Da geht es um rezeptive und produktive Texte im DaF-Unterricht, man hört, 
liest, spricht, und schreibt Texte in Deutsch. Die Arbeit mit Texten während des Sprachenlernens entwickelt Lesemotivation, Lesekompetenz und danach Schritt für Schritt die Sprachkompetenzen.

Bei der DaF-Lehrerausbildung an der Pädagogischen Fakultät der Helwan Universität in Ägypten wird das vierjährige Studium in acht Semester geteilt. Die Studierenden werden fachwissenschaftlich, pädagogisch, erziehungswissenschaftlich, fachdidaktisch und kulturell ausgebildet. Das Studium ist in Form von Vorlesungen und Schulpraktikum aufgebaut. Das umfasst 247 theoretische und praktische Wochenstunden während der vier Studienjahre. Nach der Absolvierung haben die Absolventen viele Möglichkeiten, Deutsch als Fremdsprache an Schulen zu unterrichten, und den Beruf als Lehrer auszuüben.

An Hand des Studiums werden unterschiedliche Texte vom ersten Semester bis zum achten Semester behandelt. Die Studierenden sollen fähig sein, den Text als sprachliche Einheit zu rezipieren, zu bearbeiten und bzw. zu produzieren.

Wegen der Wichtigkeit des Textes beim Lernen der deutschen Sprache versucht der folgende Beitrag, eine evaluierte Studie über die Texte und die Textauswahl in der DaFLehrerausbildung durchzuführen. Die Studierenden haben die Auswahl der Texte analysiert, auch ihre Textkompetenzen beurteilt. Deshalb haben die Studierenden im vierten Studienjahr im Jahr 2017 / 2018 ihre Meinungen über die Texte und Textauswahl während des Studiums geäußert. Sie hatten Gelegeheit, sich über alle gelesenen Texte auszudrücken, weil sie nach allen Semestern einen guten Überblick über das Studium geben können. Die Studierenden sollen in der Lage sein, Differenzen zwischen den Textsorten wahrzunehmen und sie zu vergleichen. Das würde ihnen dann helfen, wenn sie Lehrer sind. Die Studierenden sollen die Auswahl der Texte evaluieren bzw. bewerten, das ist eines der Bedürfnisse des Lehrerberufs. Wenn sie das während des Studiums machen können, dann sind sie dazu fähig, Texte in der Schule zu bestimmen, richtig angemessen auszuwählen, zu analysieren und zu bewerten. 
Darüber können sie ihre Textkompetenzen im Lichte der angebotenen Texte einschätzen und die Textkompetenzen ihrer Schüler berücksichtigen.

Dieser Beitrag zielt darauf ab, die Textauswahl aus der Sicht der Studierenden zu evaluieren, und den Zusammenhang zwischen Textauswahl und den Textkompetenzen der DaFStudierenden zu berücksichtigen, weiterhin die Kriterien für die universitäre Textauswahl in der DaF-Lehrerausbildung zu formulieren. Diese Kriterien beschreiben den Rahmen für Texte an Hand der Bildungsziele der Lehrerausbildung, des Lehrerberufs im DaF-Unterricht und der Textkompetenzen. Es ist wichtig, einige Konsequenzen für den Umgang mit Texten und mit der Textauswahl zu ziehen und den Zusammenhang zwischen Textauswahl und Textkompetenzen zu erkennen. Das wirkt auf die Lesemotivation und die Persönlichkeit der Studierenden sowie auf die Tätigkeiten des Lehrerberufs aus.

An Hand der Textkompetenzen lesen die Studierenden die Texte und nutzen alle Medien, um verschiedene Textsorten zu lesen und zu verstehen, Danach können sie über das Gelesene nachdenken oder die erworbenen Kenntnisse für andere herstellen bzw. an sie weitergeben.

Die Ergebnisse der Studierenden werden niedergeschrieben, diagnostizert und bewertet. Ferner wird der Versuch unternommen, einige Kriterien für die Texte und die Textauswahl in der universitären DaF-Lehrerausbildung vorzuschlagen. Die Ergebnisse der Studierenden leisten einen Beitrag zur Evaluation. Sie sollen in der Lage sein, das Studium, das Programm, den Inhalt, auch die Lehrkräfte auszuwerten. Ihre Einstellungen und Selbsteinschätzungen gelten als ein wichtiger Bestandteil der Qualiltätssicherung und Akkrediterung, Unterstützung und Verbesserung des Studiums.

Um diese evaluierte Studie $\mathrm{zu}$ führen, versucht die vorliegende Arbeit, auf die folgenden Fragen zu antworten:

1. Wie sieht die Textauswahl in der universitären DaFLehrerausbildung aus der Sicht der Studierenden an der Helwan Universität aus? 
2. Wie schätzen die DaF-Studierenden ihre Textkompetenzen an Hand der ausgewählten Texte ein?

3. Welche Textauswahlkriterien sollen gestärkt bzw. in den Mittelpunkt gestellt werden?

\section{2- Texte und ihre Funktionen im DaF-Unterricht}

Der Text ist ein wichtiger Bestandteil im DaF-Unterricht. Der Text gibt nicht nur Informationen für die Studierenden, sondern mehr als Informationen wie Befehle, Anweisungen, Steuerung und Emotionen (vgl. Maderova 2011: 11). Texte im DaF-Unterricht können auch Interessen erwecken. Durch unterschiedliche Texte wird der Unterricht interessanter. Der Text im Unterricht öffnet verschiedene Wege und Methoden im DaF-Unterricht. Texte werden als Quellen für Meinungsäußerungen betrachtet, weil sie auch Gelegenheiten schaffen können, mögliche Lösungen für alle Probleme zu finden, die im Text vorkommen. Der Text trägt auch zum Kommunikationsprozess bei (vgl. Davodova 2015: 8). Texte verbinden sich auch mit sozialem Handeln bei der Kommunikation (vgl. Knapp 2015: 25).

Im DaF-Unterricht werden die Texte gelesen, dialogisch nachgestaltet, darüber gesprochen und geschrieben. Die Texte haben eine zertrale Funktion nämlich als Mitteilung. Dadurch wird die Ausdrucksfähigkeit der Studierenden gefördert. Das bedeutet, dass der Begriff „Text“ viele Schritte enthält, die im Unterricht geübt werden sollen. An Hand des Begriffs „Text“ wird schon verstanden, dass Texte multifunktionalen Charakter haben (vgl. ebd: 26).

\subsection{Funktionen der Texte im DaF-Unterricht}

Der Text dient für die Studierenden verschiedenen Funktionen. Man kann die Funktionen der Texte nach der jeweiligen Textsorte bestimmen. Je nach der Textsorte werden die Funktionen unterschiedlich sein. Die Texte regen die Motivation und Interesse der Studierenden an. Texte können 
auch den Lernerfolg überprüfen, weil Texte Kontrollfunktionen bewahren (vgl. ebd 26-27). Solche Funktionen der Texte werden Schritt für Schritt im DaF-Unterricht zur Textbearbeitung erfüllt.

Die literarischen Texte haben z. B. emtionale Funktionen, sie entfalten auch die kommunikative Kompetenz der Studierenden, in unterschiedlichen Kategorien der kommunikativen Kompetenz (vgl. Jasöva 2009: 13). Die Texte beeiflussen normalerweise die Persönlichkeit der Studierenden. Deshalb werden Interesse, Bedürfnisse und Erfahrungen der Studierenden berücksichtigt, wenn man Texte auswählt (ebd. 2009: 17).

Im Literaturunterricht können Texte bestimmte Lernziele erreichen. Der Literaturunterricht bemüht sich um Ziele wie z. B., Föderung der Lesefreude, Texterschließungskompetenz, literarische Bildung, Förderung von Imagination und Kreativität, Identitätsfindung und Fremdverstehen und schließlich die Auseinandersetzung von anthropologischen Grundfragen (vgl. Wahrl / Klat / Pfister 2010: 1). Die Texte können sich positiv und negativ durch die Funktionen auf den Adressaten auswirken. Die authentischen Texte eröffnen auch eine Chance, am Alltagsleben teilzunehmen, weil diese Texte alle Informationen authentisch widerspiegeln können (vgl. Ferling 2008: 62).

Die Funktionen der Texte verbinden sich mit Textsorten. Nach (Davidova 2015: 26) werden fünf Funktionen von Texten unterschieden; die Folgenden werden genannt; Informationen z. B; Nachricht, Bericht - Apell z. B; Wahlaufruf, Kommentar Obligation z. B; Selbstverpflichtung - Kontakt - Deklaration.

Um die Textfunktionen im DaF-Unterricht zu reflektieren, sollen die Studierenden einige Fragen zu den Texten erstellen, diese Fragen fassen die möglichen Ziele und Funktionen von Texten im DaF-Unterricht zusammen.

Die Beschäftigung mit Texten kann didaktische Funktionen aufweisen. Bei der Textarbeit hat der Lehrer Gelegenheit, einen Blick auf grammatische Strukturen, Wortschatz, Redewendungen, Aussprache und Rechtschreibung zu werfen. Die Texte 
konzentrieren sich im Allgemeinen auf Lernzwecke (vgl. Davidova 2015: 50). Die Textarbeit an verschiedenen Textsorten im DaF-Unterricht wie an literarischen Texten, Sachtexten und Gebrauchenstexten entfaltet der Kritikfähigkeit (vgl. Fischer 2009: 28).

Zusammengefasst haben die Texte unterschiedliche Funktionen im DaF-Unterricht. Es ist wichtig, variierte Texte im Unterricht zu bearbeiten. Sie spielen wirkungsvolle Rollen und realisieren mehrere Ziele, die am Ende Lernerfolg und Progression im Lernprozess ermöglichen können (vgl. Moderova 2011: 18). Die Behandlung von unterschiedlichen Texten führt Schritt für Schritt zur Aufbau der Textkompetenzen, weil dies als Aufgabe der Textlinguistik gilt. Die Textlinguistik beschäftigt sich mit den textkonstituierenten Elementen, und bietet viele Möglichkeiten an, neue Wege zum Sprachgebrauch auszuprobieren (Willkop 2003: 84-106). Texte können eine zentrale Rolle bei einem Kommunikationsprozess spielen.

\subsection{Textkompetenzen als Lernziel}

Textkompetenzen ermöglichen die Studierenden schrittweise, individuell und selbstständig mit Texten $\mathrm{zu}$ arbeiten (vgl. Figl 2014: 4). Die Textkompetenzen verfügen über Schemata, Strategien und Techniken, um Kommunikationen zu erweitern (vgl. Sieber 2005: 397).

Ein Ziel des DaF-Unterrichts ist die Förderung der Textkompetenz. Anhand von Aufgaben kann man das realisieren. Die Aufgaben der Textkompetenzen beziehen sich auf Lesen, Verstehen und Produzieren von Texten. Die Studierenden entwickeln ihre Fertigkeiten, über Texte zu kommentieren. Sie können ihr Vorwissen nutzen und mit dem neuen Wissen verbinden. Dadurch bauen sie neue Strukturen (SchmölzerEibinger 2018: 11). Im DaF- Unterricht stehen die Sprachfertigkeiten „Hören, Lesen, Sprechen und Schreiben) im Zusammenhang. Die Textkompetenzen zu entwickeln, hilft den Studierenden dabei, nach dem Lesen und Verstehen von Texten, die erworbenen Kenntnisse zum Sprachgebrauch beim Sprechen und Schreiben zu nutzen. Die Lernenden sind in der Lage, Texte 
$\mathrm{zu}$ lesen $\mathrm{zu}$ verstehen, sich mündlich und schriftlich auszudrücken (Juliussen 2015: 30).

Der Text wirkt positiv bzw. negativ auf den DaF-Unterricht. Der Lese- und Schreibprozesse bei der Textrezeption und Textproduktion basieren u.a. auf dem Denken und Handeln der Studierenden. Die Lese- und Schreibprozesse dienen zur Entwicklung und zum Aufbau von Textkompetenzen im Unterricht.

Die Fokussierung von Textkompetenzen erfolgt durch einige Prozesse und Aufgaben. Die Prozesse und Aufgaben werden im Folgenden zusammengefasst;

- Veränderungen am Text,

- Strategienvielfalt und Perspektiven Wechsel,

- Fokussierung des Kernthemas,

- Bedeutungskonstruktion im Kontext,

- Themenentfaltung und Textköhärenz,

- Sprachliche Variation (vgl. Schmölzer 2018: 6-8).

Diese Prozesse und Aufgaben lassen die Studiereden rezeptiv und produktiv mit Texten umgehen; auf eine bestimmte Art und Weise wird der Text im DaF-Unterricht erfassbar und nutzbar. Diese Prozesse werden klar gezeigt in Ein-Drei-SäulenModell der Schreibförderung (vgl. Sieber 2005: 394). Dieses Modell umfasst drei Hauptprozesse nämlich (Schreibprozesse, Überarbeitungsprozess und Beurteilungsprozesse).

Es ist wichtig ein Profil für die Studierenden anzubieten, welche Textsorten lesen sie? Welche Textkompetenzen erwerben sie? Welche Probleme beim Leseunterricht haben sie? Oder welche Probleme treten beim Lesen auf? Die DaFLehrkräfte können die Nutzung der Textsorten mit einigen Lernzielen verbinden. Das hängt davon $a b$, ob Texte die folgenden Punkte im Unterricht implizieren können oder nicht. Durch die Bearbeitung von Texten im DaF-Unterricht können die gestellten Fragen nach der Thematik beantwortet werden, Fragen nach der graphischen, syntaktischen, semantischen und 
pragmatischen Textkonstitution beantwortet werden. Die Antwort auf solche Fragen garantiert den Erwerb der Textkompetenz im DaF-Unterricht. Die Antworten auf alle diese Fragen soll der Lehrer untersuchen und mit Studierenden darüber diskutieren. Diese sind ein Teil der Textkompetenzen im DaF-Unterrricht.

Die Texte im DaF-Unterricht sollen varrieren und verschiedene Textsorten repräsentieren. Die Studierenden sollen auch Kenntnisse haben über Texte und Textsorten, und Textfunktionen. Diese Kenntnisse helfen und dienen dazu, passende Texte in den DaF- Unterricht einzubetten und die sprachlichen Kompetenzen zu entwickeln (vgl. Venohr 2007: 205 nach Schönfellner 2013: 18).

\subsection{Kriterien der Textauswahl im DaF-Unterricht}

Der Lehrer soll immer die folgenden Fragen im Leseunterricht bzw. im Fremdensprachenunterricht insbesonders stellen: Welche Texte sollen die Studierenden lesen? Sind manche Textsorten geeignete für die Studierenden als andere? Welche Texte sollen die Studierenden im Unterricht bzw. außerhalb des Unterrichts lesen? Sind einige Textsorten schwieriger als andere? Die Darstellung dieser Fragen zu Beginn des Unterrichts erleichtert danach die Bearbeitung und den Umgang mit Texten. Die Studierenden sind auch positiv im Unterricht, sie sind nicht nur Empfänger, sie sollen einige Fragen zu den Texten erstellen, diese Fragen fassen die möglichen Ziele und Funktionen von Texten im DaF-Unterricht zusammen und ergänzen die Fragen des Lehrers. Einige Fragen sind wie z. B.;

Berührt der Text ein Problem? Bietet der Text Anlass zum Handeln? Hat der Text einen Sinn? Enthält der Text wissenswerte Informationen? Spricht der Text die Emotionen der Studierenden an? Hat der Text Wirkung auf die Leser oder nicht? (vgl. Gibitz 2003: 4).

Die Antworten auf die oben genannten Fragen öffnen neue Wege, um das Lernen der Sprache zu verbessern. Das führt dazu, dass die Texte auch eine didaktische Funktion haben. Der 
Umgang mit Texten gibt dem Lehrer eine Gelegenheit, einen Blick auf grammatische Struktur, Wortschatz, Redewendungen, Aussprache und Recht-schreibungen zu werfen. (vgl. Davidova 2015: 50).

Knapp (2015: 27) beschreibt die Merkmale von Textsorten und erklärt, dass die Textsorten an konkrete Realisationsformen von Texten gebunden sind. Diese Gruppe von Formen (Texten) hat bestimmte typische Merkmale und erreicht einige Lernziele.

Alle Texte wirken sich auf die Studierenden auf verschiedene Art und Weise aus. Die Anwendung von unterschiedlichen Texten im DaF-Unterricht soll auch bewertet werden (vgl. ebd. 25). In wie weit sind die Texte und Textsorten wirksam? In wie weit ist die Arbeit mit Texten sinnvoll? Die Auseinandersetzung mit dem Text schafft bestimmte Absichten $\mathrm{z}$. B; dadurch können die Studierenden den Text erleben, darüber nachdenken, und ihre Gedanken und Gefühle austauschen (vgl. Palotas 2015: 94).

Die Studierenden im universitären DaF-Unterricht beurteilen Texte, ob sie leichte oder schwierige Texte sinnvolle oder unsinnvolle Texte sind. Wenn die Texte als leichte Texte bezeichnet werden, können die Studierenden mit dem Text leicht umgehen. Choi (2002: 559) ist auch der Meinung, dass die schwierigen Texte einen Einfluss auf die Studierenden haben. Sie ermutigen sie, unterschiedliche Erfahrungen $\mathrm{zu}$ erleben. Die Auswahl von literarischen Texten im DaF-Unterricht hebt zum Beispiel die Entfaltung der Kreativität von Studierenden hervor, und das gilt als ein wichtiger Grund, um solche Textsorte auszuwählen (vgl. Jasöva 2009: 13). Jeder Text hat Ziele und befestige Absichten. Neben den verschiedenen Zwecken der Texte und Absichten des Autors beeinflussen Texte den Lehrerberuf und Persönlichkeit des Lehrers und der Studierenden. Deshalb soll die Auswahl der Texte auch den methodischen didaktischen Prinzipien entsprechen (vgl. Davidova 2015: 34).

Die Auswahl der Texte beim Lernen verfolgt einige Kriterien. Es gibt verschiedene Kriterien für die Textauswahl, die 
im Unterricht berücksichtigt werden soll. Der Lehrer im DaFUnterricht kann mögliche Kriterien umsetzen. Im Folgenden werden die verschiedenen Textauswahlkriterien dargestellt.

„Der Text als Ganzes liefert Informationen, Meinungen, Einstellungen Sachverhalte und Argumente ..... u.s.w., die im Umgang mit Texten verstanden werden müssen, um damit die Basis für den fremdsprachlichen Unterricht zu schaffen Andererseites eröffnet ein Text eine weitere Problematik, die den Sprachlernenden gelöst werden muss." (Knapp 2015: 26). Die Texte müssen Interessen der Schüler entsprechen, dem didaktischen Ziel des Unterrichts, Möglichkeiten des Unterrichts wie zeitlichen und materiellen.

Die Arbeit mit den Texten im Unterricht braucht zuerst genaue Bestimmtung von Texten, die der Lehrer anwendet. Maderova 2011 bespricht die Kriterien der Textualität zuerst, um zu bestimmen, ob sie Texte sind oder nicht. Sie ist der Ansicht, wenn eines dieser Kriterien fällt, dann handelt es sich dabei um keinen Text. Deshalb sollen diese Kriterien der Textualität zuerst berücksichtigt werden. Diese Kriterien sind; (Kohäsion - Kohärenz - Intentionalität - Akzeptabiltät Informativität - Situationalität - Intertextaulität) (Maderova 2011: 10-11).

Die Texte beziehen sich auf die konkreten Lernsituationen und die Sprachkenntnisse der Studierenden. Der Lehrer berücksichtigt nicht nur die sprachliche Seite, sondern auch die andere Seiten (Ferling 2008: 63). Welche Texte sind geeigneter als die anderen? Der Lehrer soll diese Frage stellen. Der Lehrer kann sich jedes Mal mit der Auswahl der Texte sorgfältig beschäftigen. Es wird eine Gruppe von Auswahlkriterien des Textes zusammengefasst, die auch im Auge behalten werden soll, wie z. B;

- Lernbezogene Kriterien (sprachkenntnisse, Vorwissen, muttersprachliche Lesekompetenzen)

- Textbezogene Kriterien (Inhalt und Thema, Strukturen, sprachliche Gestaltung) 
- Unterrichtsbezogene

Kriterien

(Lernziele, Aufgabenstellungen) (vgl. ebd. 63).

Die Auswahlkriterien werden unterschiedlich klassifiziert, und zwar nach dem ein- und demselben Konzept. Es gibt Beispiele von Kriterien wie:

- Auswahl der Texte nach dem Lernziel der Textarbeit.

- Auswahl der Texte nach dem Alter der Studentengruppen.

- Auswahl der Texte nach den Sprachkenntnissen der Studierenden.

- Auswahl der Texte nach der Form (Maderova 2011: 14:18).

Die Textauswahl wird auch in eine andere Klassifikation eingeteilt; einige Kriterien werden für den Unterricht und für die Auswahl der Texte gestellt, sie sind:

- Unterrichtspraktische Kriterien: Dabei werden die folgenden Punkte diskutiert wie (Schwierigkeitsgrad, Lernbedingungen, und Arbeitsweisen) .

- Die andere Gruppe von Kriterien heißt kulturtherotisches Kriterium. Das führt dazu, dass der Kultur des Landes dabei mehr Rechnung getragen wird (vgl. Hebel 1980 nach Chio 2002: 559).

Der Lehrer im DaF-Unterricht soll die Texte gut auswählen. Nicht alle Texte sind geeignet für den DaF-Unterricht oder sind für alle Ziele des DaF-Unterrichts. Zum Beispiel sind die Ziele im Literaturunterricht anders als im Grammatikunterricht. Die Arbeit mit Texten im Literaturunterricht führt zur Förderung der Lesfreude, Imagination und Kreativität normalerweise außer der Entwicklung von sprachlichen Kompetenzen bzw. Textkompetnezen (vgl. Wahr / Klat / Pfister 2010: 1).

Die Studierenden können auch andere Leskompetenzen ausüben, wie die Inhalte kritisieren bzw. bewerten, auch sie können die neuen Strukturen, die im Text stehen, mit den alten Informationen verbinden und danach bauen sie neue 
Wissensstrukturen auf (vgl. Studifit 2017: 1). Es ist auch wichtig, dass die Lehr- und Lernziele im Zusammenhang mit der Förderung der Textkompetenzen stehen (vgl. Knapp 2017: 252).

Weil die Texte einen kommunikativen, einen lernbaren Charakter und einen kulturellen Charakter haben, sollen sie sorgfältig ausgewählt werden. Die Texte sollen sich auf verschiedene Bereiche beziehen. Texte geben den Studierenden die Gelegenheit, sich mit variierten Bereichen auseinanderzusetzen.Texte können auch in allen Phasen des Unterrichts integriert werden, um unterschiedliche Funktionen zu erfüllen. Darüber hinaus soll die kulturelle Seite nicht bei der Auswahl der Texte vermieden werden. Die ausgewählten kulturellen Themen im Unterricht tragen zur Förderung der interkulturellen Kompetenzen bei (Kertes 2015: 111).

Die Texte im DaF-Unterricht können dazu führen, dass die Persönlichkeiten der Studierenden entwickelt werden können. Deshalb sollen Texte die Ziele, die Interessen, Berdürfnisse, Erfahrungen, Wünsche und Weltwissen der Studierenden mit einander verknüpfen.Wichtig ist es auch, dass die Texte sowohl für die Studierenden als auch für den Lehrer kommunikationsfördend sind, weil sie Partner und verantwortlich für das eigene Lernen im Unterricht sind.

\section{Methodisches Vergehen}

Die vorliegende Arbeit verfolgt bestimmte Schritte, um das Ziel zu erreichen bzw. um die Texte in der universitären DaFLehrerausbildung an der Helwan Universität zu analysieren, zu bewerten und auch die Textkompetenzen der Studierenden einzuschätzen und schließlich die Auswahlkriterien zu erstellen. Diese Schritte sind im Folgenden beschrieben.

\subsection{Analyse der Texte in der DaF-Lehrerausbildung der Helwan Universität}

Die DaF-Studierenden während ihres Studiums bearbeiten fachliche Themen in Bezug auf das Lehren und Lernen der deutschen Sprache. Das führt dazu, dass sie ihre Kenntnisse über pädagogische und sprachliche Bereiche entwickeln können. 
Durch die Behandlung der fachlichen Bereiche werden auch berufliche Kompetenzen für den DaF-Lehrer erworben. Die Bearbeitung von fachlichen Texten bzw. Themen ist sinnvoll für geeignete Ausbildung und Berufswege (vgl. ebd 2015: 4).

Das Studium an der Pädagogischen Fakultät der Helwan Universität steuert die Rollen des DaF-Lehrers und bereiten die Studierenden auf Prinzipien des DaF-Unterrichts vor. Die Lehrenden haben im Laufe der Arbeit an Schulen eine diagnostische Aufgabe. Sie nehmen an der Schulentwicklung durch Entdecken der Stärken und Schwächen des Lernprozesses teil. Sie haben auch eine Aufgabe nämlich Materialien und Lerntexte auszuwählen, damit sie Lernerfolg erreichen können. Die DaF-Lehrenden bemühmen sich darum, Texte und Textwelten mit den Lebenswelten der Lernenden zu verbinden. Das schafft einerseits Interesse und Motivation im Unterricht und andererseits Lernfortschritt und Lernprogression (vgl. Knapp 2015: 34).

Die DaF-Lehrerausbildung lässt die Studierenden unterschiedliche Textsorten kennen lernen. Das Studium enthält insgesamt 241 Stunden in acht Semesten, das dauert vier Jahre. 94 Stunden sind für Lektüre und andere Fächer, die textorientiert bzw. textzentriert sind. (Anhang 1)

Die Studierenden arbeiten mit Texten direkt und indirekt. Das heißt; sie bearbeiten Texte, sie lesen, verstehen und entwickeln ihre Lesekompetenzen direkt in den Fächern, die sich mit Lesen und Lesekompetenzen als Haupziel im Unterricht beschäftigen wie im Fach Lektüre. Sie arbeiten aber mit Texten indirekt, wenn das Ziel etwas anderes ist, außer Lesekompetenzen zu entfalten, nämlich das Lesen als mittelere Fertigkeit wie im Fach Aufsatz oder Fach Grammatik.

Die folgende Tabelle stellt die Fächer, in denen die Studierenden während des Studiums mit Texten arbeiten können. Dadurch werden die Lesekompetenzen durch Hören, Lesen, Sprechen und Schreiben entwickelt. Die Studierenden trainieren, wie sie Texte reziptieren und produzieren, d. h., besprechen, interpretieren, bewerten und kommentieren. Ich 
habe die Fächer im Folgenden genannt, die eine direkte Beziehung zu den Texten haben.

Tab. 1:Stundenplan für die im Zusammenhang mit dem Lesen stehenden Fächer

\begin{tabular}{|c|c|c|c|}
\hline \multicolumn{2}{|c|}{$\begin{array}{l}\text { Das erste Studienjahr } \\
\text { Erstes Semester }\end{array}$} & \multicolumn{2}{|c|}{$\begin{array}{l}\text { Das erste Studienjahr } \\
\text { Zweites Semester }\end{array}$} \\
\hline Fächer & Stunden & Fächer & Stunden \\
\hline Lektüre 1 & 5 & Lektüre 2 & 4 \\
\hline Sprachübungen 1 & 6 & Sprachübungen 2 & 4 \\
\hline \multicolumn{2}{|c|}{$\begin{array}{l}\text { Das zweite Studienjahr } \\
\text { Erstes Semester }\end{array}$} & \multicolumn{2}{|c|}{$\begin{array}{c}\text { Das zweite Studienjahr } \\
\text { Zweites Semester }\end{array}$} \\
\hline Fächer & Stunden & Fächer & Stunden \\
\hline $\begin{array}{c}\text { Lektüre und schriftlicher } \\
\text { Ausdruck }\end{array}$ & 4 & Übung zur Textanalyse & 4 \\
\hline Literaturwissenschaft & 4 & $\begin{array}{c}\text { Sprachübungen und } \\
\text { schriftlicher Ausdruck }\end{array}$ & 4 \\
\hline Kultur & 4 & $\begin{array}{l}\text { Lernstrategien der } \\
\text { deutsche Sprache }\end{array}$ & 4 \\
\hline \multicolumn{2}{|c|}{$\begin{array}{l}\text { Das dritte Studienjahr } \\
\text { Erstes Semester }\end{array}$} & \multicolumn{2}{|c|}{$\begin{array}{c}\text { Das dritte Studienjahr } \\
\text { Zweites Semester }\end{array}$} \\
\hline Fächer & Stunden & Fächer & Stunden \\
\hline Übersetzung & 4 & Analyse epischer Texte & 4 \\
\hline $\begin{array}{l}\text { Überblick über die } \\
\text { literarischen Epochen }\end{array}$ & 4 & $\begin{array}{c}\text { Sprachliche } \\
\text { Kommunikation und } \\
\text { Aufsatz } \\
\end{array}$ & 4 \\
\hline Allgemeine Methodik & 2 & $\begin{array}{c}\text { Das deutsche } \\
\text { Bildungssystem in } \\
\text { Deutschland }\end{array}$ & 4 \\
\hline \multicolumn{2}{|c|}{$\begin{array}{l}\text { Das vierte Studienjahr } \\
\text { Erstes Semester }\end{array}$} & \multicolumn{2}{|c|}{$\begin{array}{c}\text { Das vierte Studienjahr } \\
\text { Zweites Semester }\end{array}$} \\
\hline Fächer & Stunden & Fächer & Stunden \\
\hline Spezifische Methodik & 3 & $\begin{array}{c}\text { Kultur und Landeskunde: } \\
\text { Problemfelder deutscher } \\
\text { Kultur }\end{array}$ & 4 \\
\hline $\begin{array}{c}\text { Interpretation von } \\
\text { dramatischen und lyrischen } \\
\text { Texten }\end{array}$ & 5 & Übersetzung 2 & 5 \\
\hline Lektüre und Aufsatz & 4 & $\begin{array}{c}\text { Literarische Texte und } \\
\text { Stilanalyse }\end{array}$ & 4 \\
\hline $\begin{array}{c}\text { Kultur und Landeskunde } \\
\text { interkulturelle } \\
\text { kommunikation }\end{array}$ & 4 & & \\
\hline Gesamte Stunden & 49 & Gesamte Stunden & 45 \\
\hline
\end{tabular}

Fächern bearbeitet. Die Studierenden hören und lesen Texte, erschließen Hauptideen, sammeln Informationen, interpretieren 
Gedanken, geben Zusammenfassungen, analysieren und kommentieren die Texte. Sie können endlich viele Leseziele erreichen.

Alle oben genannten Fächer haben enge Beziehungen $\mathrm{zu}$ den deutschen Texten. Die Studierenen sollen bei der Auswahl von Texten während des Studiums eine Rolle spielen. Die Lehrkräfte im universitären Unterricht sollen den Studierenden die Möglichkeit teilweise geben, selbst Lesetexte auszuwählen. Ihre Auswahl der Texte eröffnet große Wege zum Selbstlernen, Selbstvertrauen und danach Selbstbewerten. Die DaFStudierenden sollen diese Kompetenzen erwerben, weil sie solche Kompetenzen danach als DaF- Lehrende in Schulen ausüben werden. Sie werden vermitteln, was sie gelernt haben und wie sie es gelernt haben. Deshalb ist es wichtig, dass sie sich daran gewöhnen, einen Beitrag im Unterricht und im Lernprozess zu leisten.

\subsection{Auswertung der Textauswahl aus der Sicht der Studierenden}

Es ist wichtig, die Einstellungen der Studierenden zum Gelernten zu analysieren. Die Entwicklung von Lernprogrammen für die universitäre Lehrerausbildung hängt auch von den Einstellungen der Studierenden ab. Sie sollen Veränderungen bewirken. Wenn sie ihre Meinungen immer äußern, beteiligen sie sich an der Entwicklung.

\subsubsection{Aufbau der Befragung}

Ich habe eine Befragung entwickelt, um die Einstellungen der Studierenden zu den gelernten Texten zu erkennen. Ich habe durch diese Befragung viele Informationen über universitäre Texte an der Helwan Universität gesammelt. Das Ziel war, dass die Studierenden die Texte bewerten, und ihre Meinungen ausdrücken können. Ich habe die Meinungen und die Einstellungen der Studierenden im vierten Studienjahr 2017/2018 analysiert, sie waren 35 Studierende. Die Befragung besteht aus 16 Fragen; 7 Fragen werden als geschlossenen Fragen (mit JA/NEIN) bezeichnet und 9 Fragen werden als 
offenen Fragen bezeichnet, in denen die Studierenden frei über Texte und Textauswahl geäußert haben. (Anhang 2)

\subsection{Selbesteinschätzung der Textkompetenzen}

Nach der Realisierung des Fragebogens zur Textauswahl war ich sehr interessiert zu wissen, wie die Textkompetenzen bei den DaF-Lehrerstudierenden aussehen. Die Selbsteinschätzung der Studierenden zu den Textkompetenzen wird gefördert, damit die DaF-Studierenden zunächst ihre Textkompetenzen feststellen und dann beurteilen können. Dadurch kann ich ein Profil über den Zusammenhang zwischen Textauswahl und den Textkompetenzen aufbauen. Eine gezielte Textauswahl für die DaF-Studierenden an der Lehrerausbildung hat ihre Wirkung auf die Entwicklung der Textkompetenzen. Die Lehrkräfte, die die Textkompetenzen der Studierenden entwickeln wollen, können diesen Zusammenhang zwischen Textauswahl und Textkompetenzen berücksichtigen.

\subsubsection{Befragung der Selbsteinschätzung}

Ich habe einen Fragebogen zur Selbsteinschätzung der Textkompetenzen bei den DaF-Studierenden entwickelt. Nach der Formulierung des Fragebogens will ich überprüfen, ob der Fragebogen reliabel oder nicht ist, um anzugehen, ob ich mich auf die Ergebnisse der Selbsteinschätzung verlassen kann. Das Kerterium Reliabilität bezieht sich auf die Zuverlässigkeit und Genauigkeit eines Tests.

Ich habe die Re-Test-Reliabilität verwendet. Ich gebe den DaF-Studierenden (Testgruppe), die 20 Studierende in diesem Fall waren, einen Fragebogen (Selbsteinschätzung) an einem Tag und nach einer Woche wird der Fragebogen wiederholt. Daraus ergibt sich, dass die Zuverlässigkeit des Fragebogens der Selbsteinschätzung an Hand des Korrelationskoeffizierten nach Pearson 0.70 ist. Die Ergebnisse zeigen, dass der Fragebogen reliabel ist, und die Ergebnisse möglichst wenige Messfehler haben.

Die Befragung umfasst vier Kompetenzfelder der Textkompetenzen. Diese Felder sind wie folgt dargestellt; 
Kompetenzfeld 1 : ein allgemeines Verständnis des Textes entwickeln

Kompetenzfeld 2 : explizite Informationen ermitteln

Kompetenzfeld 3: eine textbezogene Kommunikation entwickeln

Kompetenzfeld 4: strategisches Denken entwickeln und den Inhalt des Textes reflektieren und bewerten. (Anhang 3)

\section{Die Ergebnisse}

Die Studierenden haben auf die Fragen der Befragungen geantwortet. Die Antworten geben einen Überblick über die Beschreibungen von Leseunterricht und die Behandlungen von Texten in der DaF-universitären Lehrerausbildung an der Pädagogischen Fakultät der Helwan Universität und die Textkompetenzen werden auch bewertet.

\subsection{Ergebnisse der Befragung der Textauswahl 4.1.1 Ergebnisse der offenen Fragen}

Ich präsentiere zuerst die Antworten der DaF-Studierenden auf die 9 offenen Fragen:

Welche beliebten Texte lesen Sie? Die Antworten zeigen uns den Kenntnisstand der Studierenden über die Textsorten, welche Textsorten sie erkannt, bearbeitet, und auch ihnen gefallen haben. Die Studierenden haben verschiedene Textsorten wie folgt ausgewählt; $63 \%$ der Studierenden wählen "Kurzgeschichte“ als die beliebteste Textsorte. $49 \%$ der Studierenden lesen aber Aufsätze. $43 \%$ der Studierenden wählen Nachrichten. $40 \%$ der Studierenden lesen aber Kindergeschichte, 34 \% der Studierenden wählen Anweisungen. $30 \%$ der Studierenden wählen Märchen. 23 \% der Studenten Romane. Diese Frage lässt die Studierenden mehr als eine Antwort bzw. Textsorte auswählen.

Es ist wichtig, die Meinungen der DaF-Studierenden über das Lesen und den Lernprozess zu berücksichtigen. Sie sollen sich auch selbst fragen, warum sie lesen? in wie weit das Lesen für die eigene Persönlichkeit für das Leben und für den zukünftigen Beruf notwendig ist?. Die Studierenden 
antworten folgendermaßen, Lesen macht sie glücklich/ froh/ zufrieden. Durch Lesen bekommen sie Informationen. Durch Lesen erleben sie viele Erfahrungen. Durch Lesen verbesseren sie ihre Kenntnisse. Wenn sie lesen, ist die Zeit spannend und interessant. Durch lesen erwerben sie neue Wörter, um Gespräche führen zu können. Wenn sie lesen, sind sie neugierig. Durch lesen verbesseren sie ihre Sprache und ihre Aussprache. Durch Lesen erkennen sie die Kultur der deutschsprachigen Länder. Durch Lesen wird die Kommunikation verbessert. Das Lesen gibt ihnen Gelegenheit, ihre Fehler zu korrigieren. Sie überprüfen immer ihre Lesefähigkeit.

Eine Frage betrifft auch die jeweiligen Texte. Die Studierenden haben Gelegenheit, ihre Meinung über die gelesenen Texte zu äußern. Sie finden Texte schwer bzw. wichtig fürs Leben, oder interessant und nützlich, andere Texte aber langweilig und unwichtig.

Eine Frage bezieht sich auf Themen; welche Themen die Studierenden lesen möchten. Sie geben die folgenden Antworten:

- Lerntypen und Lehrerrolle im Unterricht.

- Freundschaft.

- Mode und Schönheit.

- Gesellschaftliche Phänomene.

- Geschichte der Länder und die deutsche Geschichte.

- Fakten, Kultur, Berufsleben, Sport und aktuelle Themen.

- Wirtschaft und Politik.

- literarische Texte (Romane - Kurzgeschichte

- Kindergeschichte - moderne Literatur).

- Traditionen (Sitten und Gräusche).

- Lehrerberuf.

- Umwelt.

- Kunst.

- Umgang mit Konflikten. 
- Probleme der Schüler und Probleme in der Schule.

Wenn diese Frage gestellt wird, welche Themen auf den Lehrerberuf auswirken können, sie haben z. B. gesagt; Themen über Rolle des Lehrers im Unterricht - Lernertypen - Lehr-und Lernstrategien - Lehr- und Lernmethoden - Grammatik im Unterricht - Kultur - Schulsystem - Ausbildung - Lernstoff vermitteln - Kinderunterricht - Lebenslanges Lernen Praktikum - nichts. Einige Studierende haben auf diese Frage nicht geantwortet. Sie nennen also in welchen Fächern, sie die oben genannten Themen behandelt haben. Wie z. B. Fach Methodik - Fach Literatur - Fach Landeskunde - Fach Lektüre Fach Aufsatz - Fach Kultur - Fach Übersetzung - Fach Sprachübungen. Das heißt, einige Fächer beziehen sich auf den Lehrerberuf und Lehrerausbildung bzw. Lehrerkompetenzen.

Es ist auch klar, dass die Rolle des sozialen Lernens effektiv ist. Das soll an Stelle der Einzelarbeit treten und im Mittelpunkt stehen. Die Studierenden antworten auf die Frage „Wie sollte gelesen werden", dass sie einen Überblick zuerst erhalten und dann Informationen erschließen wollen. Sie mögen Feadback, Rückmeldungen und Hinweise nach dem Lesen vom Lehrer bekommen. Ferner möchten sie Texte durch Medien und visiuelle Mittel lesen.

Eine Frage lautet, Welche Aktivitäten, Lesetechniken üben sie beim Lesen aus?

Sie notieren folgende Antworten; Notizen machen - Lesen in Gruppen - Markieren - Zusammenfassungen machen - Filme Sehen - Diskussion über Texte führen - Rollen spielen Karteikarten erstellen - Kommentare geben - Aufsätze schreiben.

Die Studierenden äußern ihre Wünsche über Texte, die sie lesen möchten. Sie haben die Möglichkeit, mehrere Aussagen zu wählen. Sie äußern ihre Meinungen wie folgt: $74 \%$ der Studierenden möchten lustige und spannende Texte. $69 \%$ der Studierenden möchten fantastische Texte. $34 \%$ der Studierenden möchten lehrreiche Texte. 31 \% der Studierenden 
möchten informative Texte. $23 \%$ der Studierenden möchten romantische Texte.

\subsubsection{Ergebnisse der geschlossenen Fragen}

Um die Antworten der DaF-Studierenden auf die geschlosenen Fragen $\mathrm{zu}$ analysieren, habe ich das statistische SPSS Programm angewendt.

Ergebnisse der geschlossenen Fragen (Frage 1 - Frage 5 Frage 6 - Frage 7 - Frage 8 - Frage 14 - Frage 15)

Tab 2: Die Ergebnisse der Studierenden in Median und Prozent

\begin{tabular}{|c|c|c|}
\hline & Median & Ergebnisse in \% \\
\hline Frage 1 & 2.17 & 54.29 \\
\hline Frage 5 & 0.46 & 45.71 \\
\hline Frage 6 & 0.80 & 80 \\
\hline Frage 7 & 0.20 & 20 \\
\hline Frage 8 & 0.60 & 60 \\
\hline Frage 14 & 1.89 & 62.86 \\
\hline Frage 15 & 0.31 & 31.43 \\
\hline Total & 6.43 & 53.57 \\
\hline
\end{tabular}

Die Tabelle zeigt die Unterschiede zwichen den Antworten auf die Fragen.

Die Frage 1 lautet: Wie oft lesen Sie deutsche Texte?

Die DaF-Studierenden haben darauf geantwortet. Die folgende Tabelle zeigt die Unterschiede der Antworten von Studierenden. Die Antworten haben 5 Möglichkeiten (immer regelmäßig - oft - selten - niemals). Die Antworten werden (von 4 bis null) klassifiziert. Die Antwort („immer" bekommt 4) und („niemals“ bekommt null).

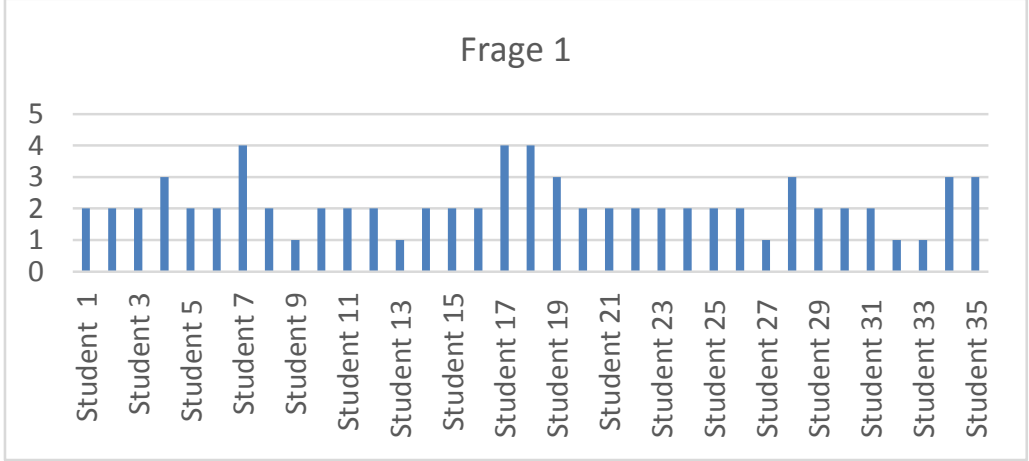

Abb.1: Die Unterschiede der Antworten der Studierenden auf Frage1 
Die Abbildung zeigt, dass nur 3 Studierende darauf reagieren, dass sie immer deutsche Texte lesen.

Die Frage 5 lautet: Haben die Texte, die Sie gelernt haben, einen Bezug zum Lehrerberuf?

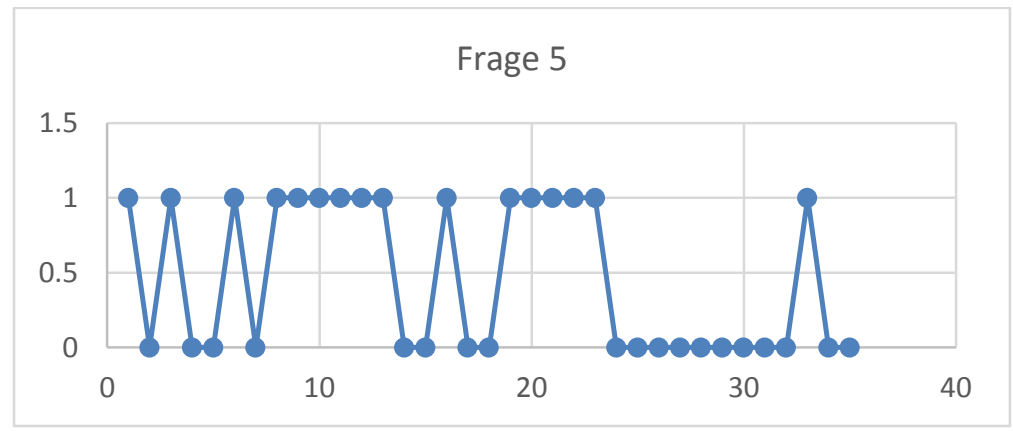

\section{Abb. 2:Die Unterschiede der Antworten der Studierenden auf} Frage5

Die Antworten auf diese Frage haben nur (1 für JA/ und Null füe Nein). Es wird gezeigt, wer Ja sagt und wer Nein sagt. Die Studierenden, die die Texte keinen Bezug zum Lehrerberuf finden, sind mehr als die anderen Studierenden, die dafür sind.

Frage 6 lautet : Enthalten die gelesenen Texte wichtige wissenswerte Informationen?

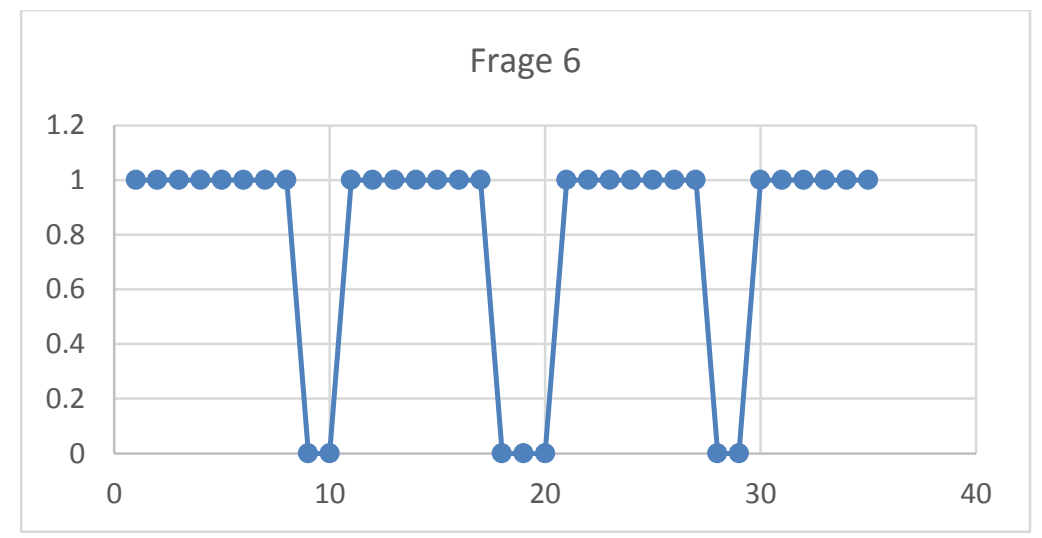

Abb. 3: Die Unterschiede der Antworten der Studierenden auf Frage6 
Die Antworten auf diese Frage haben nur (1 für JA/ und Null füe Nein). Es wird gezeigt, wer Ja sagt und wer Nein sagt. Die Antworten erklären, dass die gelesenen Texte im universitären Unterricht wichtige Informationen enthalten,

Die Frage 7 lautet: Sprechen die gelernten Texte Ihre Emotionen an?

\section{Frage 7}

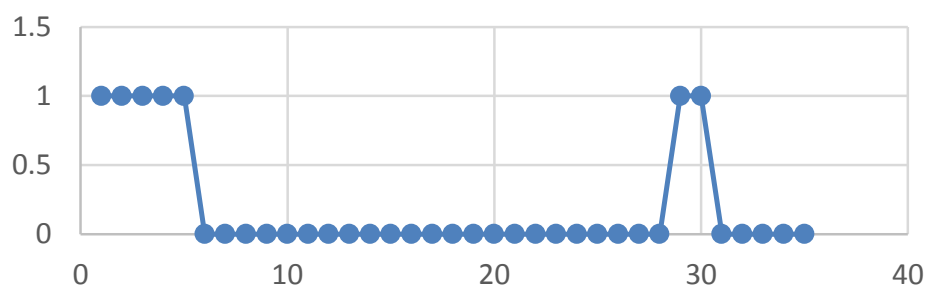

Abb. 4: Die Unterschiede der Antworten der Studierenden auf Frage 7

Die Antworten auf diese Frage haben nur (1 für JA/ und Null für Nein). Es wird gezeigt, wer Ja sagt und wer Nein sagt. Die Antworten zeigen, dass Texte der Emotionen der Studierenden nicht ansprechen.

Die Frage 8 lautet : Haben die gelesenen Texte eine Auswirkung auf Ihr Leben?

\section{Frage 8}

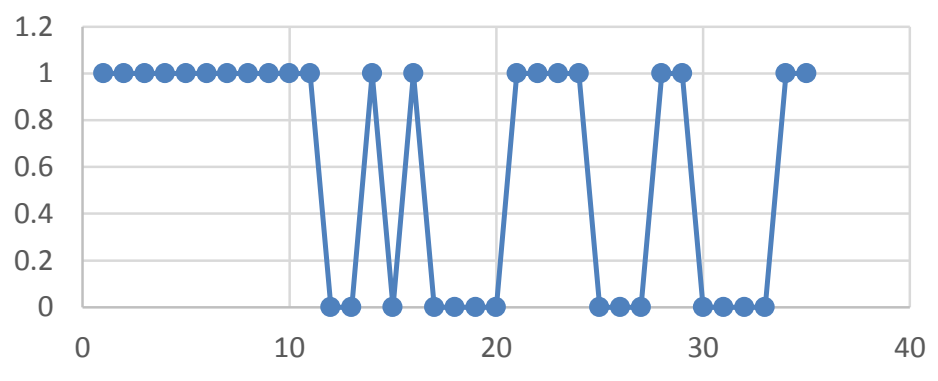

Abb. 5: Die Unterschiede der Antworten der Studierenden auf Frage8 
Die Antworten auf diese Frage haben nur (1 für JA/ und Null füe Nein). Es wird gezeigt, wer Ja sagt und wer Nein sagt. Diese Antworten zeigen, dass Texte eine Wirkung auf das Leben der Studierenden haben.

Die Frage 14 lautet: Wie möchten Sie im Unterricht lesen, alleine/ in Partnerarbeit/ oder in Gruppenarbeit und warum?

\section{Frage 14}

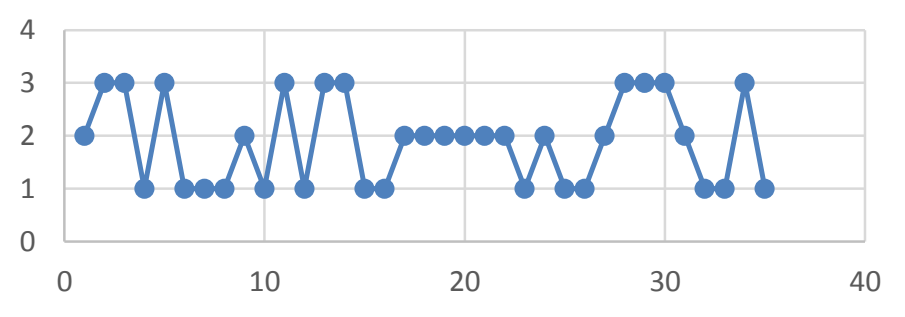

\section{Abb. 6: Die Unterschiede der Antworten der Studierenden auf}

\section{Frage14}

Die Antworten auf diese Frage haben (3 Punkte für Gruppenarbeit / 2 Punkte füe Partnerarbeit / und 1 Punkt für Einzelarbeit). Die Antworten haben gezeigt, wie viele Studierende Gruppenarbeit, Partnerarbeit und Einzelarbeit auswählen. Die Studierenden sollen lernen, wie sie mit einander besser lesen können und die Texte bearbeiten können, und ihre Erfahrungen austauschen können.

Die Frage 15 lautet : Haben Sie einmal Texte zum Lesen ausgewählt?

Frage 15

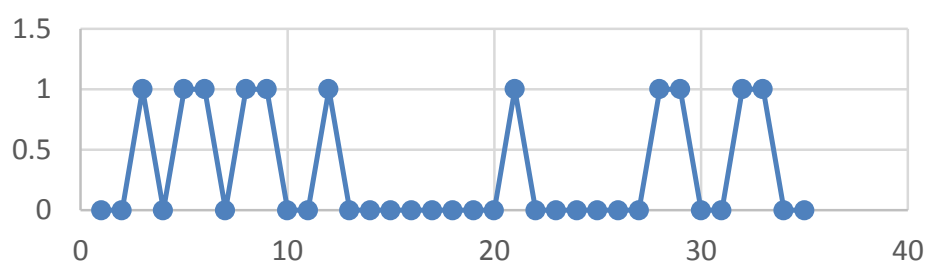

Abb. 7: Die Unterschiede der Antworten der Studierenden auf Frage15 
Die Antworten auf diese Frage haben nur (1 für JA/ und Null für Nein). Es wird gezeigt, wer Ja sagt und wer Nein sagt. Die Studierenden haben Möglichkeiten, einige Texte auszuwählen, aber diese Möglichkeit ist auch beschränkt.

Alle Antworten der DaF-Studierenden an der Helwan Universität gelten als genaue Kritik an Texten, Textauswahl und Textarbeit. Die Studierenden haben sich mit Texten kritisch auseinandergesetzt. Die Antworten haben bei der Formulierung der Kriterien der Textauswahl geholfen. Die Antworten geben noch ein genaues Profil der Texte, das bezeichnet auch die Stellung des Lesens in der DaF-Lehrerausbildung. Das führt dazu, dass der Textauswahl in der universitären DaF-Lehrerausbildung mehr Rechnung getragen wird.

Ich kann auch an Hand der Ergebnisse die folgenden Punkte zusammenfassen:

- Die Studierenden lesen deutsche Texte, aber sie brauchen Aufmerksamkeit auf Leseziele und Textsorten.

- Die Studierenden bekommen auf verschiedene Art und Weise Informationen von Texten, was auch förderlich ist, Fertigkeiten und Strategien zu erwerben.

- Die Emotionen der Studierenden sollen bei der Textauswahl betrachtet werden.

- Die Lehrkräfte können die Teilnahme der Studierenden an der Textauswahl berücksichtigen.

\subsection{Ergebnisse der Befragung der Selbsteinschätzung}

Die Ergebnisse $\mathrm{zu}$ den Textkompetenzen zeigen die Beurteilung der Textkompetenzen von den DaF-Studierenden. Nicht nur Studierende können über ihre Selbsteinschätzung reflektieren, sondern auch die Lehrkräfte an der Pädagogische Fakultät der Helwan Universität.

Tab 3: Die Antworten auf die Selbsteinschätzung der DaFStudierenden in unterschiedlichen Kategorien

\begin{tabular}{|c|c|c|c|c|c|}
\hline & Feld 1 & Feld 2 & Feld 3 & Feld 4 & Totale \\
\hline Mittelwert & 2.88 & 3.00 & 2.83 & 2.64 & 67.1 \\
\hline Median & 17.50 & 16.00 & 16.50 & 19.00 & 69.00 \\
\hline Ergebnisse in \% & 72.08 & 75.00 & 70.63 & $\mathbf{6 6 . 0 0}$ & $\mathbf{6 9 . 9 0}$ \\
\hline
\end{tabular}

Die Tabelle 3 zeigt die Unterschiede der Antworten auf die Selbsteinschätzung der Textkompetenzen in verschiedenen 
Kategorien wie Mittelwert, Medien und Ergebnisse in \% für jedes Kompetenzfeld und für die gesamten Antworten. Die DaFStudierenden haben ihre Textkompetenzen bewertet. Die Totalen Ergebnisse in \% betragen 69.90, die auch erklären, welche Textkompetenzen weitere Entwicklung und Aufmerksamkeit im universitären DaF-Unterricht brauchen. Kompetenzfelder können nach den Ergebnissen geordnet werden wie z. B.; Kompetenzfeld 2, Kompetenzfeld 1, Kompetenzfeld 3 und Kompetenzfeld 4. Die Ergebnisse der Befragung zeigen auch, dass die DaF-Studierenden das Niveau des Könnens nicht erreicht haben; (Niveau des Könnens wird in dieser Arbeit in $80 \%$ bestimmt).

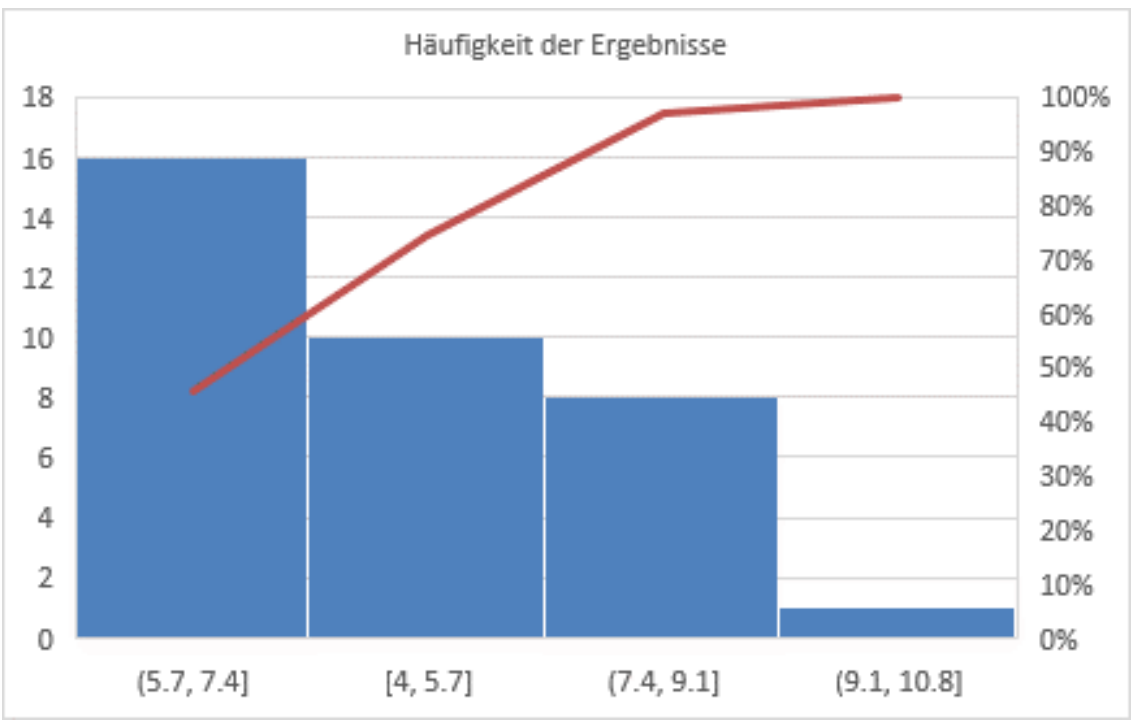

Abb. 8: Die Häufigkeiten der Ergebnisse für alle Textkompetenzen

Die Abbildung zeigt die Haufigkeiten der Antworten der Studierenden in absteigender Reihenfolge für die 4 Felder der Textkompetenzen, die ich bestimmt habe. Kompetenzfeld 4 braucht mehr Ausüben im Unterricht, damit die DaFStudierenden diese Textkompetenzen erwerben können. 
Median der Studierenden für Feld 1

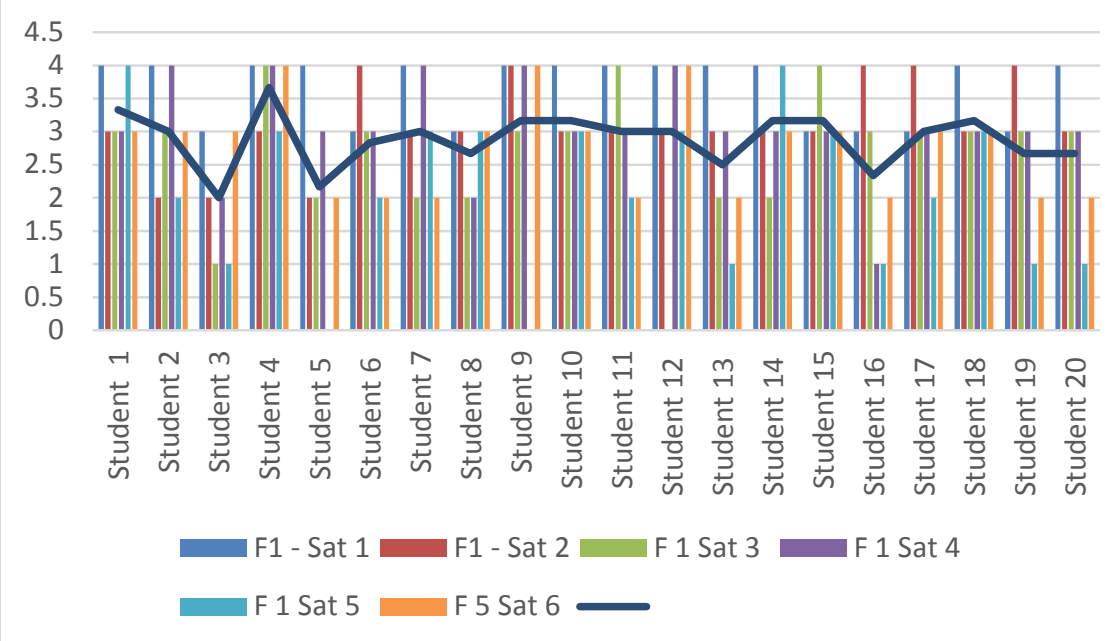

Abb. 9: Unterschiede der Median für Kompetenzfeld 1 : ein allgemeines Verständnis des Textes entwickeln

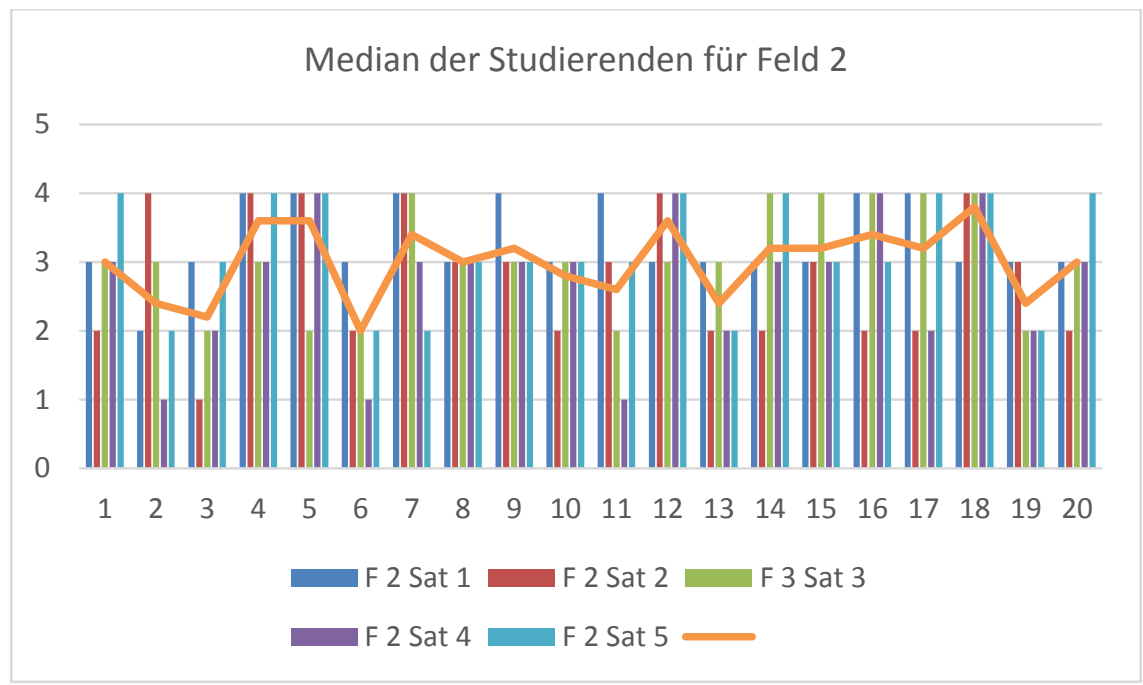

Abb. 10: Unterschiede der Median für Kompetenzfeld 2 : explizite Informationen ermitteln 


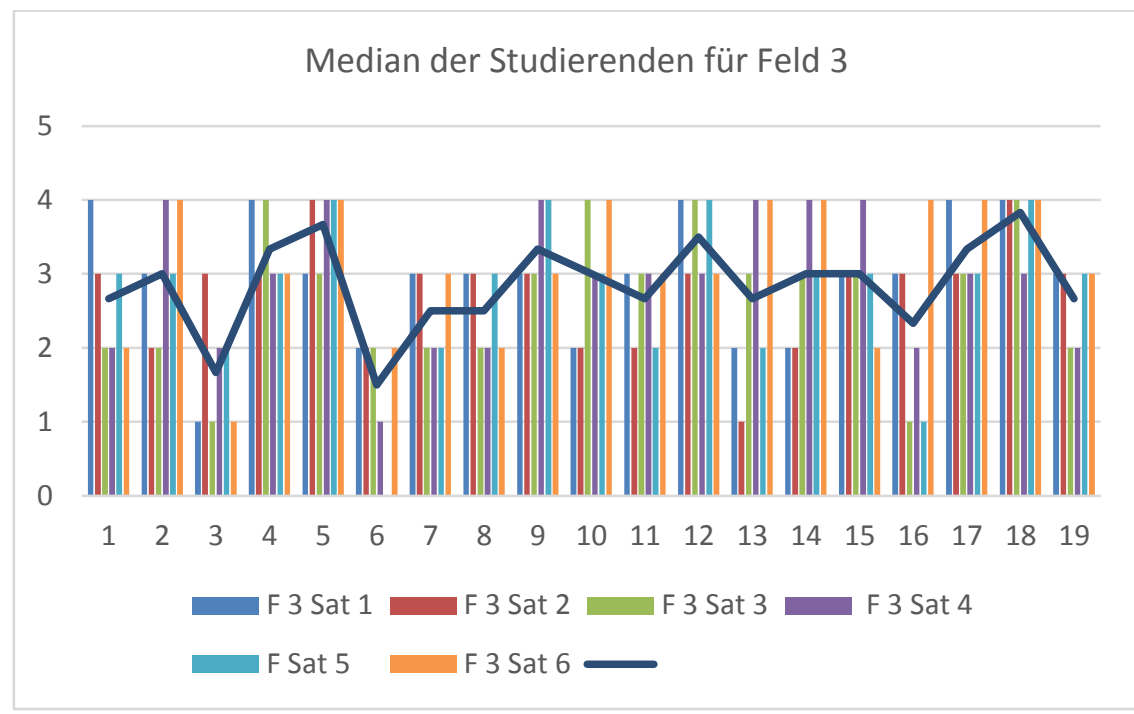

Abb. 11: Unterschiede der Median für Kompetenzfeld 3 : eine textbezogene Kommunikation entwickeln

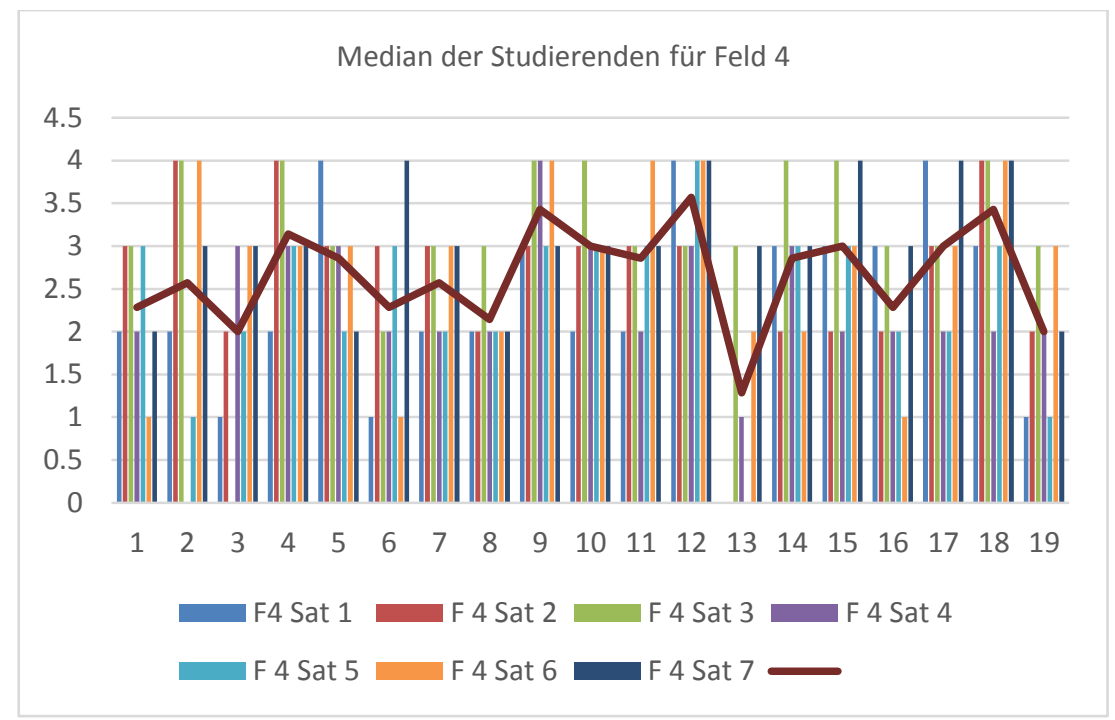

Abb. 12: Unterschiede der Median für Kompetenzfeld 4 :strategisches Denken entwickeln und den Inhalt des Textes 


\section{JRCIET}

\section{Unterschiede der Median für alle Textkompetenzen}

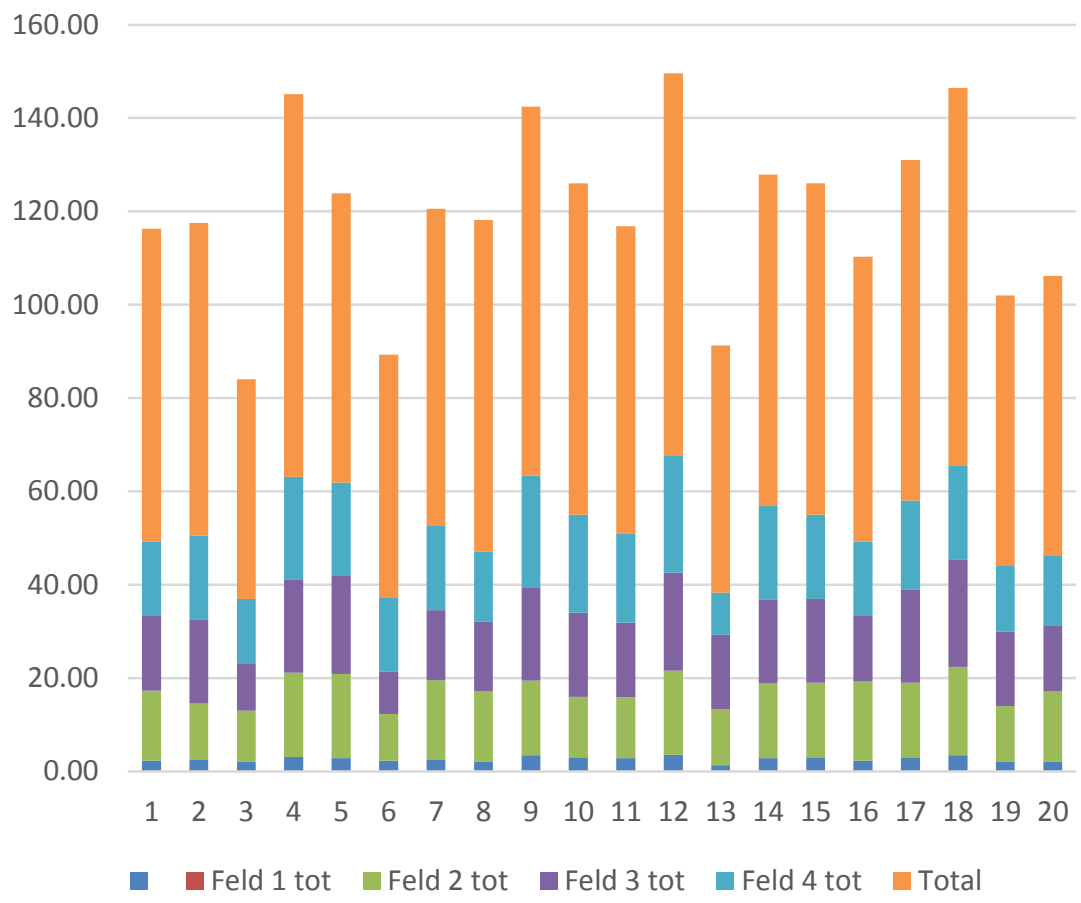

Abb. 13: Unterschiede der Median für Textkompetenzen

Durch die Beurteilung der Textkompetenzen ergeben sich die folgenden Punkte:

- Es besteht ein Zusammenhang zwichen Text und Entwicklung der Textkompetenzen.

- Die DaF-Studierenden sollen zunächst die Texte lesen und verstehen, dann bearbeiten, darüber nachdenken und zum Sprachgebrauch nutzen.

- Die Lehrkräfte können alle Einzelheiten der Textkompetnezen im DaF-Unterricht berücksichtigen.

- Wenn die Lehrkräfte Texte im Unterricht beringen, sollen sie diese Texte nach einigen Kriterien auswählen. 


\section{3 vorgeschlagene Kriterien für Texte im universtären DaF-Unterricht}

Die DaF-Lehrerausbildung soll sich mit notwendigen Textorientierungen im Unterricht beschäftigen. Dabei sollen Texte im Lichte einiger Kriterien ausgewählt und bearbeitet werden. Die vorliegende Arbeit bezieht sich darauf, Kriterien für Texte und Textauswahl im universitären DaF-Unterricht an Hand der Untersuchung der Studenteneinstellungen und der Selbsteinschätzung.

Ich habe fünf Auswahlkriterien bestimmt. Sie Sind:

Kriterium 1 : Bildungsziele und Kompetenzen

Die Bildungsziele und Kompetenzen werden unter Brücksichtigung des Studiums und der Dauer des Studiums formuliert.

Kriterium 2: Studenteninteresse

Studenteninteresse habe ich an Hand der Einstellungen der Studierenden gesammelt und notiert.

Kriterium 3: Inhalt und Thema

Inhalt und Thema sind auf GER und das Lernen der deutschen Sprache bezogen.

Kriterium 4: Lehrerberuf

Lehrerberuf steht im Vordergrund in der DaFLehrerausbildung. Texte sollen dem Lehrerberuf entsprechen.

Kriterium 5 : Textkompetenzen

Die Textkompetenzen stehen im Mittelpunkt des Fremdsprachenunterrichts und gewinnen an Bedeutung. Deshalb findet ein Zusammenhang zwischen Textauswahl im Unterrricht und Textkompetenzen statt. Textauswahl kann bei der Entwicklung von Textkompetenzen helfen.

Wenn die Lehrkräfte in der universitären DaFLehrerausbildung die Texte im Unterricht bringen, sollen sie die Texte unter Brücksichtigung der Kriterien auswählen. Der folgende Kriterienvorschlag kann dabei helfen, Texte im Bezug auf Studierenden, ihre Interessen und ihren zukünftigen Lehrerberuf auszuwählen. 
Tab. 4:Kriterienvorschlag der Textauswahl für die DaFLehrerausbildung an der Pädagogischen Fakultät

\begin{tabular}{|c|c|c|c|c|}
\hline & \multicolumn{4}{|c|}{ Textauswahlkriterien für die DaF-Lehrerausbildung } \\
\hline & \begin{tabular}{|l|} 
Bildungsziele \\
\end{tabular} & $\begin{array}{l}\text { Studenten- } \\
\text { interesse }\end{array}$ & $\begin{array}{l}\text { Inhalt } \\
\text { Thema }\end{array}$ & Lehrerberuf \\
\hline $\begin{array}{l}\text { Das erste } \\
\text { Studienjahr }\end{array}$ & $\begin{array}{l}\text { Vermittlung von } \\
\text { sprachlichen } \\
\text { Kompetenzen }\end{array}$ & $\begin{array}{l}\text { Spannende } \\
\text { Texte } \\
\text { Lustige } \\
\text { Texte } \\
\text { Lehrreiche } \\
\text { Texte } \\
\text { Informative } \\
\text { Texte } \\
\text { Romantische } \\
\text { Texte }\end{array}$ & $\begin{array}{l}\text { Lektüre } \\
\text { Einfache } \\
\text { Texte } \\
\text { Themen: } \\
\text { Familien - } \\
\text { Freundschaft } \\
\text { - Mode - Sport } \\
\text { - Umwelt - } \\
\text { Kunst - Berufe } \\
\text { - tägliches } \\
\text { Leben }\end{array}$ & $\begin{array}{l}\text { Texte stellen den } \\
\text { Lehrerberuf vor, } \\
\text { und beziehen sich } \\
\text { auf } \\
\text { Lehrerberuf. }\end{array}$ \\
\hline $\begin{array}{l}\text { Das zweite } \\
\text { Studienjahr }\end{array}$ & $\begin{array}{l}\text { Förderung und } \\
\text { Unterstützung der } \\
\text { Sprachverwendung } \\
\text { Vermittlung von } \\
\text { kommunikativen } \\
\text { Kompetenzen }\end{array}$ & $\begin{array}{l}\text { Spannende } \\
\text { Texte } \\
\text { Lustige } \\
\text { Texte } \\
\text { Lehrreiche } \\
\text { Texte } \\
\text { Informative } \\
\text { Texte } \\
\text { Romantische } \\
\text { Texte }\end{array}$ & $\begin{array}{l}\text { Sachtexte } \\
\text { Themen: } \\
\text { Deutsche } \\
\text { Geschichte - } \\
\text { Wirtschaft - } \\
\text { Politik - } \\
\text { Berufsleben - } \\
\text { Sitten und } \\
\text { Traditionen - } \\
\text { Sprache }\end{array}$ & $\begin{array}{l}\text { Texte steuern den } \\
\text { Lehrerberuf und } \\
\text { Rolle des Lehrers } \\
\text { in der } \\
\text { Gesellschaft. }\end{array}$ \\
\hline $\begin{array}{l}\text { Das dritte } \\
\text { Studienjahr }\end{array}$ & $\begin{array}{l}\text { Vermittlung der } \\
\text { beruflichen } \\
\text { professionellen } \\
\text { Kompetenzen }\end{array}$ & $\begin{array}{l}\text { Spannende } \\
\text { Texte } \\
\text { Lustige } \\
\text { Texte } \\
\text { Lehrreiche } \\
\text { Texte } \\
\text { Informative } \\
\text { Texte } \\
\text { Romantische } \\
\text { Texte }\end{array}$ & $\begin{array}{l}\text { Fachtexte } \\
\text { Literarische } \\
\text { Texte } \\
\text { Themen: } \\
\text { Lehrerberuf - } \\
\text { Rolle des } \\
\text { Lehrers - } \\
\text { Kultur } \\
\text { Globalisierung } \\
\text { - Lernen und } \\
\text { Lernen uation } \\
\text { Evaluaten }\end{array}$ & $\begin{array}{l}\text { Texte erstellen } \\
\text { Probleme beim } \\
\text { Lehrerberuf und } \\
\text { entdecken } \\
\text { Lösungen. Wia } \\
\text { verhählt sich der } \\
\text { Lehrer } \\
\text { unterschiedlichen } \\
\text { Problemen? }\end{array}$ \\
\hline $\begin{array}{l}\text { Das vierte } \\
\text { Sudienjahr }\end{array}$ & $\begin{array}{ll}\text { Förderung } & \text { und } \\
\text { Unterstützung } & \text { der } \\
\text { sozialen, } & \\
\text { kulturellen } & \text { und } \\
\text { persönlichen } & \\
\text { Kompetenzen } & \\
\end{array}$ & $\begin{array}{l}\text { Spannende } \\
\text { Texte } \\
\text { Lustige } \\
\text { Texte } \\
\text { Lehrreiche } \\
\text { Texte } \\
\text { Informative } \\
\text { Texte } \\
\text { Romantische } \\
\text { Texte }\end{array}$ & $\begin{array}{l}\text { Komplizierte } \\
\text { Texte } \\
\text { Literarische } \\
\text { Texte } \\
\text { Themen: } \\
\text { Kultur von } \\
\text { Ägypten und } \\
\text { Deutschland - } \\
\text { Heimatland } \\
\text { Arbeit und } \\
\text { Zukunft und } \\
\text { Erfolg un } \\
\text { personliche } \\
\text { Entwicklung - } \\
\text { Eigenschaften } \\
\text { der anderen - } \\
\text { menschliche } \\
\text { Beziehungen }\end{array}$ & $\begin{array}{l}\text { Texte entwickeln } \\
\text { sich } \\
\text { Persönlichkeit des } \\
\text { Lehrers. } \\
\text { Texte steuern die } \\
\text { Zukunftpläne, } \\
\text { lebenslanges } \\
\text { Lernen und } \\
\text { Peerlernen. und }\end{array}$ \\
\hline rium & & & & \\
\hline
\end{tabular}




\section{Fazit:}

Die DaF-Lehrerausbildung an der pädagogischen Fakultät der Helwan Universität bemüht sich, die Ausbildung zukünftiger DaF-Lehrer an Vorbereitungs- und Oberschulen zu optimieren, damit sie Deutsch als Fremdsprache in den Schulen mit qualifizierten professionellen Kompetenzen unterrichten können. Die Studierenden können den Erwerb von Wissen, Kompetenzen und Strategien bei der Sprachverwendung nutzen.

Texte spielen eine wichtige Rolle im DaF-Unterricht und stehen im Zusammenhang mit Sprachkompetenzen insbesondere mit Textkompetenzen. Eine Folge davon sollen Texte sorgfältig ausgewählt werden. Die Textauswahl hängt von verschiedenen Kriterien ab. Deshalb versucht diese Arbeit, die Anforderungen, die an einen Text gestellt werden, zu optimieren und die Textqualität in der universitären DaF-Lehrerausbildung zu verbessern. Die Studierenden sollen auf eine bestimmte Art und Weise am Unterricht teilnehmen. Ihre Teilnahme hat eine große Bedeutung. Sie haben in diesem Beitrag ihre Meinung dazu geäußert. Ihre Einstellungen zum Leseunterricht, zu Lesetexten und -themen helfen bei der Formulierung der Textauswahlkriterien. Diese Kriterien werden unter Berücksichtigung des Fachstudiums in der DaFLehrerausbildung aufgebaut.

\section{Literaturangaben}

Choi, Young Jin (2002): Literatur im universitären DaF-Unterricht in Korea. Zur Verzahnung von Theorie und Praxis. In InfoDaF 29 (6), 555-566.

Davidova, Zdenka (2015): Texte und Textverstehen im Fremdsprachenunterricht.Online:

https://is.muni.cz/th/uha4v/DIPLOMOVA_PRACE_Davidov a_Z..pdf [22.04.2017].

Gibitz, Urlich (2003): Handlungsorientierte und authentische Textarbeit im Fremdsprachenunterricht (am Beispiel des Deutsch als Fremdsprache in Kamerum), Goethe Institut Yaounde, Online: www.goethe.de/af/yao[18.07.2017]. 
Ferling, Nikola (2008): Lesen im DaZ Unterricht In: Kaufmann, Susan u.a. 2008: Fortbildung für Kursleitende Deutsch als Zweitsprache, Band 2, Didaktik Methodik, Hueber Verlag, GmbH\&Co.KG. Ismaning, Deutschland, (Seiten 35-67).

Figl, Ursula (2014): Textkompetenz und Sprachbildung in allen Gegenständen, Ein Modell zur Untersetzung an Weiner AHS mit Schwerpunkt Sek1, Standschulrat für Wien, Pädagogische Hochschule Wien.

Fischer, Christian (2009): Texte, Gattungen, Textsorten und ihre Verwendung in Lesebüchern, Dissertation zur Erlangung des Doktorgrades der Philosophie des Fachbereiches Sprache, Literatur und Kultur der Justus-Liebig-Universität Gießen, Gießen.

Jasöva, Marcela (2009): Arbeit mit literarischen Texten im DaFUnterricht am Beispiel eines Textesvon christine Nöstlinger, Diplomarbeit. Masaryk Universität, Pädagogische Fakultät, Lehrstuhl für Deutsche Sprache und Literatur, Brünn.

Juliussen, Reidun Oline Holme (2015): Zur Entwicklung von Textkompetenz anhand digitaler Hilfsmittel, Masterarbeit, Universität Bergen.

Kapp, Ilona Feld (2015): Deutsch als Fremdsprache (DaF) in Ungarn, ein Überblick über den DaF-Unterricht und seine Erforschungen In: Boōcz, Barna Katalin u.a. 2015: Deutsch als Fremdsprache Unterrichten lernen, Eötvös Loränd Tudomanyegyetem, ELTE, Budapest.(Seiten 23-39). Online:http://metodika.btk.elte.hu/file/TAMOP_BTK_BMK_ 6pdf.[4.12.2017].

Knapp, Ilona Feld (2017) : Textkompetenzen und Aufgabenorientierung im Fremdsprachenunterricht. In: Freundenberg-Findeisen Renata (Hrsg) http://www. academia.edu/31718681/Textkompetenz_und_Aufgabenor ientierung_im_Fremdsprachenunterricht_In_FreudenbergFindeisen_Renate_Hrsg._(Seiten 245-256)- [23.07.2017].

Kertes, Patricia (2015): Textarbeit im DaF-Unterricht. Der Weg vom Text zum Text im Fokus, Die reflektierte Unterrichtspraxis. In: Boōcz, Barna Katalin u.a. 2015: 
Deutsch als Fremdsprache Unterrichten lernen, Eötvös Loränd Tudomanyegyetem, ELTE, Budapest.(Seiten 108134).

Online:

http://metodika.btk.elte.hu/file/TAMOP_BTK_BMK_6.pdf.[ 4.12.2017].

Madervoa, Kristina (2011): Textarbeit im Fremdunterricht, Einfache Lektüre im Deutschunterricht, Diplomarbeit, Masaryk Universität, Pädagogische Fakultät, Lehrstuhl für Deutsche Sprache und Literatur, Brünn.

Palotäs, Berta (2015): Der Weg vom Text zum Text im Fokus, die sprachlichen Mittel: In Boōcz, Barna Katalin u.a. (2015): Deutsch als Fremdsprache Unterrichten lernen, Eötvös Loränd Tudomanyegyetem, ELTE, Budapest.(Seiten 90-107). Online:http://metodika.btk.elte.hu/file/TAMOP_BTK_BMK_ 6.pdf.[4.12.2017].

Schmölzer- Eibinger, Sabine (2018): Textkompetenz, Lernen und literale Didaktik.www.abrapa.org.br/cd/npdfs/schmoelzer Eibinger-sabine(17.09.2018).

Schönfellner, Sabine (2013): Der Vermittlung und Überprüfung der Schreibfertigkeit in Deutsch als Fremdsprache auf Niveau B2, Magisterarbeit, Universität Wien.

Sieber, Peter (2005): Didaktik des Schreibens vom Produkt zum Prozess und weiter zur Textkompetenz, Schweizerische Zeitschrift für Bildungswissenschaften 27 (2005) 3, 281-406, www.pedocs.de.

Stochmann, Reinhard (2004): Was ist eine gute Evaluation? Einführung zu Funktionen und Methoden von Evaluationsverfahren. Saarbrücken 2002. Zentrum für Evaluation. Online: www.ceval.de/modox/fileadmin/user upload/pdf/workpaper9.pdf.[17.2.2018].

Studifit Projekt (2017): Methoden und Techniken für die Hochschullehre. Ein Projekt an der Textarbeit HTWK Leipzig, Bundesminsterium für Bildung und Forschung unter dem Förderkennzeichen gefördert. Online: 
https://www.htwkleipzig.de/hochschule/organisationstruktur/ hochschulleitung/prorektor-bildung/studifit/Textarbeit Mut.pdf[4.12.2017 ].

Wahrl, Clandia/ Klat, Julian/ Pfister, Chrispher (2010): Methoden der Texterschließung: Rezeptiv-Analytische Verfahren/ Handlungs-und Produktionsorientierte Verfahren, Universität Bayrenth.

Willkop, Eva-Maria (2003) : Anwendungsorientierte Textlinguistik, Am Beispiel von Textsorten, Isotopien, Tempora und Referenzformen In GFL 3/2003, 84-106, Mainz. 